\title{
Contrôle de la fabrication du fromage Emmenthal par la méthode conductrimétrique
}

\author{
par \\ J. TSOULI*, A. VILLE* et H. VALLA**
}

Dans une étude préliminaire [1] nous avons montré l'intérêt que peut avoir la mesure conductimétrique dans l'appréciation du temps au bout duquel un lait coagule sous l'action d'une enzyme protéolytique tel qu'un mélange pepsine-présure ou un extrait enzymatique provenant de Mucor miehei. Nous avons laissé entrevoir les possibilités qu'offre cette méthode pour guider le fromager dans la définition de certains paramètres qui lui sont utiles tels que par exemple, le temps au bout duquel il doit travailler le caillé.

C'est dans cette optique que nous avons orienté le présent travail au cours duquel nous avons étudié l'évolution de la conductance du milieu pendant trois périodes à savoir :

- celle de l'ensemencement par des ferments lactiques au cours de laquelle le lait subit une certaine maturation,

- celle de l'emprésurage jusqu'au début de la formation du caillé,

- une période de $5 \mathrm{mn}$ au cours de laquelle le caillé évolue vers une certaine texture jugée satisfaisante pour que le fromager décide d'entreprendre les opérations ultérieures.

Ce travail a été élaboré au laboratoire avec le matériel et les techniques déjà décrits [1] sur des échantillons de lait et autres produits nécessaires provenant de fabrications d'Emmenthal en usine $^{* * *}$ qui étaient strictement contrôlées pour nous permettre de confronter nos appréciations mutuelles.

* Université Claude-Bernard, Lyon 1 ; I.U.T. 1, Département de Biologie Appliquée, 43, bd du 11 Novembre 1918, F - 69621 Villeurbanne.

Nous tenons à remercier Mme M. Gilet pour sa collaboration technique au cours de cette étude.

** Division Recherche Développement Gilca, rue de la Convention, Vienne.

*** Nous remercions les responsables des Ets Vivalp S.A., St-Laurent-sur-Saône, auprès desquels nous avons trouvé toute l'aide nécessaire pour réaliser le présent travail. 


\section{MODALITES EXPERIMENTALES}

\section{$1^{\circ}$ Produits utilisés}

- lait frais de mélange employé pour la fabrication de l'Emmenthal de $\mathrm{pH}$ variant entre 6,4 et 6,6. Les échantillons de lait sur lesquels nous avons travaillé étaient pris dans les cuves de fabrication et nous parvenaient au laboratoire dans des conditions parfaites de conservation (sous régime du froid) $2 \mathrm{~h}$ après leur prélèvement,

- présure traditionnelle liquide ayant une force coagulante de $1 / 10000$. L'échantillon de présure était prélevé sur le lot servant à la fabrication et renouvelé chaque semaine,

- présure en poudre nous permettant de faire chaque fois une coagulation avec le même paramètre enzymatique. Le dosage avec la présure en poudre est fait de telle sorte que le temps au bout duquel se produit la coagulation du lait est approximativement le même que celui obtenu avec la présure liquide, surage,

- ferments lactiques pour la maturation du lait avant empré-

- chlorure de calcium codex.

\section{$2^{\circ}$ Appareillage}

Le montage est identique à celui déjà utilisé [1] et décrit à l'occasion d'études effectuées pour suivre des cinétiques enzymatiques par conductimétrie $[2,3,4,5]$. Rappelons cependant que cette mesure est faite par l'intermédiaire d'un pont à transformateurs à courant alternatif à une fréquence de $1592 \mathrm{~Hz}$. Notons aussi que la température de travail du lait est $32^{\circ} \mathrm{C}$, celle précisément utilisée pour la fabrication de l'Emmenthal à l'usine avec laquelle nous avons collaboré.

Parallèlement à ce travail au laboratoire, nous avons également effectué un essai sur cuve à l'usine même, ce qui nous a conduit à construire par nos propres moyens une électrode similaire à celle que nous avons utilisée jusqu'à présent mais dont les dimensions correspondent à l'échelle industrielle.

\section{$3^{\circ}$ Etude expérimentale}

Le lait frais de mélange, additionné de $\mathrm{CaCl}_{2}$ Codex $(0,17 \mathrm{~g} / 1 \mathrm{de}$ lait) est introduit dans la cellule à raison de $45 \mathrm{ml}$ et y est maintenu à $32^{\circ} \mathrm{C}$ pendant toute la durée des opérations. Pour avoir une idée du rôle des différents paramètres mis en cause dans la coagulation du lait, plusieurs essais ont été réalisés :

- un premier enregistrement est obtenu seulement avec le lait additionné de $\mathrm{CaCl}_{2}$ et emprésuré à $32^{\circ} \mathrm{C}$ avec une solution enzyma- 
tique préparée à partir de la présure en poudre (utilisée comme enzyme standard) ce qui nous permet de travailler toujours dans les mêmes conditions avec une matière première différente d'un jour à l'autre,

- les $2^{\mathrm{e}}$ et $3^{\mathrm{e}}$ essais sont effectués dans les mêmes conditions que celles réalisées en usine, le lait additionné de $\mathrm{CaCl}_{2}$ est ensemencé avec des ferments lactiques ( 4 p. 100 de bactéries mésophiles et 2 p. 100 de bactéries thermophiles). Au bout de $40 \mathrm{mn}$ de maturation à $32^{\circ} \mathrm{C}$, le lait est emprésuré, dans un cas avec une solution enzymatique préparée à partir de la présure en poudre et dans l'autre cas avec une solution de la présure liquide utilisée en usine ( $25 \mathrm{cc} / 1001$ de lait) et ce de telle sorte que le temps au bout duquel on obtient la coagulation de $45 \mathrm{ml}$ de lait est approximativement le même que celui obtenu lors de la fabrication de l'Emmenthal à l'usine.

Ce travail a duré 3 mois à raison de deux à trois manipulations par semaine représentant une dizaine d'essais.

D'habitude, le fromager attend environ $5 \mathrm{mn}$ après le début de la prise du lait pour laisser le temps au caillé d'atteindre une texture aussi optimale que possible avant de procéder aux autres opérations (tranchage, chauffage, etc.) ; nous avons à notre tour poursuivi l'enregistrement de l'évolution de la conductance du caillé pendant un temps analogue pour voir si le tracé obtenu ne présentait pas des phénomènes capables de nous renseigner sur cette texture optimale à obtenir.

Le travail à l'usine s'est effectué normalement sur une cuve d'une capacité de 75001 de lait avec l'électrode spécialement conçue pour cette échelle : le profil de la courbe obtenue dans ce cas est comparable à ceux réalisés au laboratoire en utilisant la cellule de $45 \mathrm{ml}$.

Deux exemples d'enregistrements sont représentés par la figure 1.

\section{DISCUSSION}

D'après le tracé des différentes courbes nous constatons que l'adjonction de ferments lactiques au lait provoque une augmentation de la conductance du milieu ce qui est dû naturellement à une certaine augmentation de l'acidité du lait au cours de sa maturation. Ensuite, après l'emprésurage du lait on assiste pendant un certain temps à une montée régulière de la courbe due également à une diminution du $\mathrm{pH}$ du milieu de quelques fractions d'unités $\mathrm{pH}$ avant d'entamer une chute traduisant une diminution de la conductance au début de la formation des micelles. Nous avons dit [1] que ce phénomène pourrait être probablement en relation avec le rôle que joueraient les éléments ioniques du milieu dans la formation des micelles [6,7] mais en plus nous avons cherché à savoir si le 


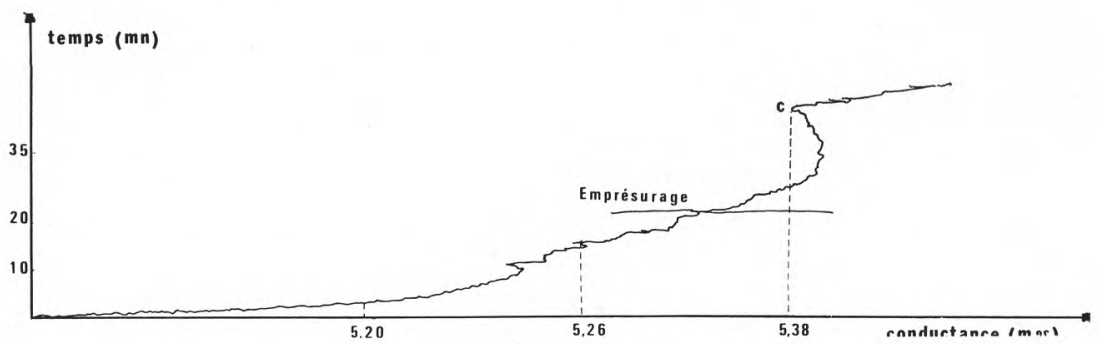

fig. 1 a

Coagulation effectuée sans addition de ferment lactique avec la présure en poudre (enzyme standard)

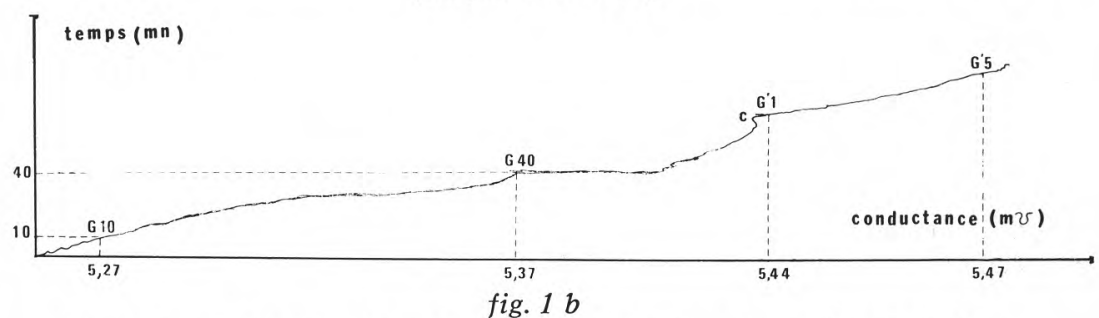

Coagulation effectuée dans les mêrnes conditions que celles de l'usine avec ferments lactiques et présure liquide

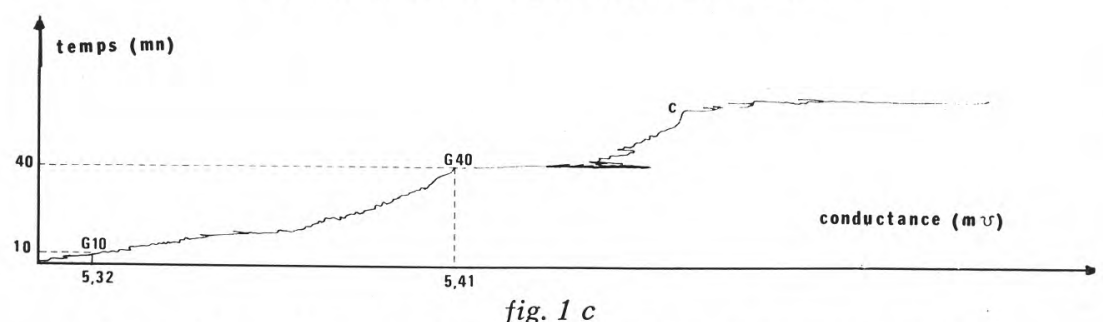

Coagulation effectuée avec ferments lactiques et présure en poudre dans les mêmes conditions que celles de l'usine

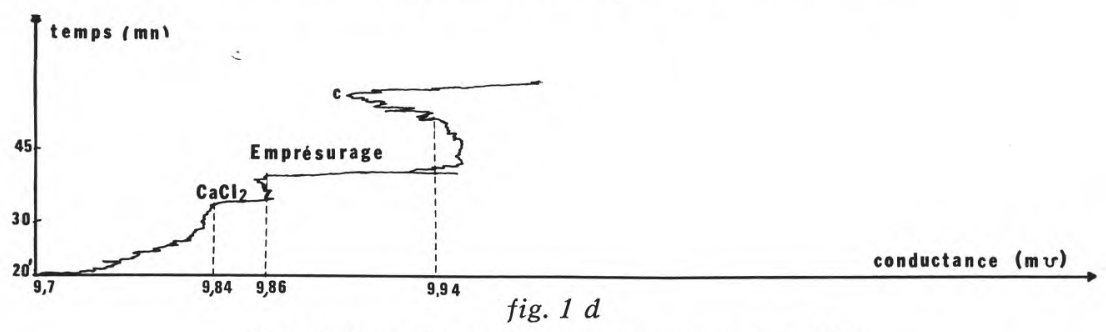

Essai effectué à l'usine sur une cuve de 75001

fig. $1^{*}$

Exemples de tracés obtenus au cours de différents essais 
changement d'état du milieu n'était pas pour quelque chose dans cette diminution de la conductance. Pour cela, nous avons procédé à l'enregistrement dans les mêmes conditions que celles du lait, de l'évolution de la conductance lors de la formation d'un gel de polyacrylamide à 5 p. 100 . Nous avons assisté au même phénomène de chute de la conductance au moment de la formation du gel et de changement de pente dans le sens d'une augmentation de cette conductance ensuite c'est-à-dire une analogie parfaite avec ce qui se passe dans le cas du lait (fig. 2).

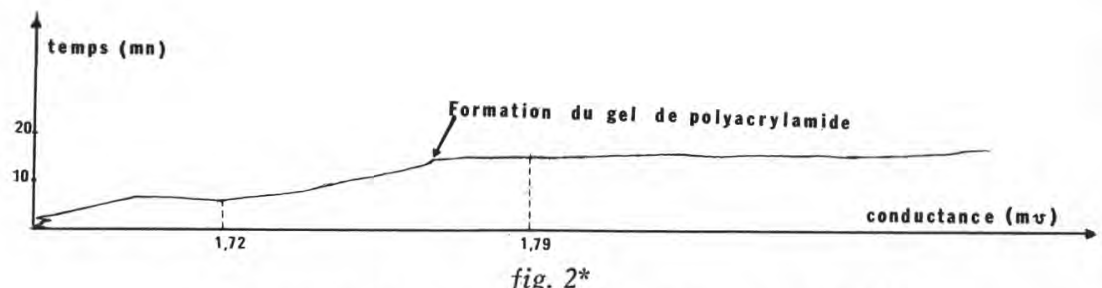

Polymérisation d'un gel de polyacrylamide à 5 p. 100

Cela nous amène à penser que la diminution de la conductance constatée lors de la formation des micelles de caséine est due non seulement aux éléments ioniques dont nous avons déjà parlé mais aussi au phénomène physique de la formation du gel (caillé) ralentissant ainsi la mobilité des ions à cause de l'augmentation de la viscosité du milieu.

Nous nous sommes préoccupés ensuite du stade de travail des ferments lactiques dans l'intervalle de temps compris entre 10 et $40 \mathrm{mn}$ après leur adjonction au lait. Le point de la courbe correspondant à $10 \mathrm{mn}$ a été choisi arbitrairement car il est indispensable d'attendre une stabilité du tracé après l'addition des ferments.

Nous avons ainsi pu suivre l'évolution de la conductance $\mathrm{G}$ du milieu entre les points $\mathrm{G}_{10}$ et $\mathrm{G}_{40}$ (fig. $1 \mathrm{~b}$ ) et nous avons calculé le

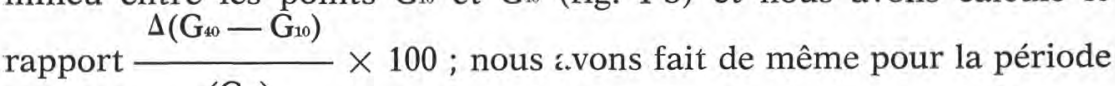
$\left(\mathrm{G}_{10}\right)$ comprise entre $1 \mathrm{mn}$ et $5 \mathrm{mn}$ (points $\mathrm{G}_{1}^{\prime}$ et $\mathrm{G}_{5}^{\prime}$ ) dans la phase de l'évolution du caillé vers la texture désirée.

Les résultats obtenus figurent dans les tableaux 1 et 2 . Nous constatons que la variation relative de conductance due au travail

* Les figures 1 et 2 sont les reproductions fidèles des enregistrements originaux réduits au $1 / 4$ à l'aide d'un photocopieur réducteur. 


\section{TABLEAU 1}

Variation de conductance au cours du travail des ferments lactiques entre $G_{10}$ et $G_{40}$ (fig. 1 b) pendant l'essai identique à ceux de l'usine

\begin{tabular}{|c|c|c|c|}
\hline Essais & $\mathrm{G}_{10}$ en millimho & $\mathrm{G}_{40}$ en millimho & $\Delta \frac{\mathrm{G}_{40}-\mathrm{G}_{10}}{\mathrm{G}_{10}} \times 100$ \\
\hline $\begin{array}{r}1 \\
2 \\
3 \\
4 \\
5 \\
6 \\
7 \\
8 \\
9 \\
10 \\
11 \\
12\end{array}$ & $\begin{array}{l}5,16 \\
5,45 \\
5,58 \\
5,30 \\
5,37 \\
5,18 \\
5,27 \\
5,26 \\
5,26 \\
5,24 \\
5,19 \\
5,26\end{array}$ & $\begin{array}{l}5,25 \\
5,59 \\
5,69 \\
5,40 \\
5,49 \\
5,28 \\
5,37 \\
5,36 \\
5,38 \\
5,38 \\
5,32 \\
5,39\end{array}$ & $\begin{array}{l}1,7 \\
2,5 \\
2 \\
1,9 \\
2,2 \\
1,9 \\
1,9 \\
1,9 \\
2,2 \\
2,7 \\
2,5 \\
2,5\end{array}$ \\
\hline & \multicolumn{2}{|c|}{ Moyenne } & $2,16 \pm 0,32^{*}$ \\
\hline \multicolumn{2}{|c|}{ *l'erreur standard $=\varepsilon=\sqrt{\frac{\varepsilon(x-\bar{x})^{2}}{n-1}}$} & \multicolumn{2}{|c|}{$\begin{aligned} x= & \text { valeur de } \Delta \frac{\mathrm{G}_{40}-\mathrm{G}_{10}}{\mathrm{G}_{10}} \times 100 \\
& \text { pour les différents essais. } \\
\bar{x}= & \text { valeur moyenne }=\frac{\varepsilon x}{\mathrm{n}} \\
& \text { n étant le nombre d'essais. }\end{aligned}$} \\
\hline
\end{tabular}

des ferments est de l'ordre de 2 p. $100(2,16 \pm 0,32)$ d'une part et d'autre part cette variation atteint 0,5 p. $100(0,47 \pm 0,09)$ dans la phase correspondant à la formation du caillé (entre les points $\mathrm{G}^{\prime}$ et $\mathrm{G}_{5}^{\prime}$ ).

Ainsi pourrait-on envisager dans le cas du contrôle de la fabrication du fromage Emmenthal par la méthode conductimétrique de noter une variation de conductance au cours du travail des ferments lactiques de l'ordre de 2 p. 100 de $G_{10}$ pour décider de l'emprésurage du lait. On suivra ensuite le profil de la courbe dû à l'action enzymatique jusqu'au point $\mathrm{C}$ correspondant au début de la formation du caillé (fig. $1 \mathrm{~b}$ ) ; il suffirait alors de suivre l'évolution de la conductance du milieu à partir de $G_{1}^{\prime}$ jusqu'à atteindre une variation de l'ordre de 0,5 p. 100 pour décider d'entreprendre les opérations ultérieures. Cela se justifierait par les concordances notées, dans l'appréciation de la qualité des différents caillés obtenus parallèlement à l'usine et au laboratoire avec la cellule de $45 \mathrm{ml}$. 


\section{TABLEAU 2}

Variation de conductance au cours de l'évolution du caillé entre $G_{1}^{\prime}$ et $G_{5}^{\prime}$ (fig. 1 b)

\begin{tabular}{|c|c|c|c|}
\hline Essais & $\mathrm{G}_{1}^{\prime}$ en millimho & $\mathrm{G}_{2}^{\prime}$ en millimho & $\Delta \frac{\mathrm{G}_{5}^{\prime}-\mathrm{G}_{1}^{\prime}}{\mathrm{G}_{1}^{\prime}} \times 100$ \\
\hline $\begin{array}{r}1 \\
2 \\
3 \\
4 \\
5 \\
6 \\
7 \\
8 \\
9 \\
10\end{array}$ & $\begin{array}{l}5,27 \\
5,78 \\
5,46 \\
5,52 \\
5,29 \\
5,45 \\
5,38 \\
5,44 \\
5,47 \\
5,47\end{array}$ & $\begin{array}{l}5,29 \\
5,81 \\
5,48 \\
5,55 \\
5,31 \\
5,47 \\
5,41 \\
5,47 \\
5,50 \\
5,50\end{array}$ & $\begin{array}{l}0,38 \\
0,52 \\
0,37 \\
0,54 \\
0,38 \\
0,37 \\
0,56 \\
0,55 \\
0,55 \\
0,55\end{array}$ \\
\hline & \multicolumn{2}{|c|}{ Moyenne } & $0,47 \pm 0,09^{*}$ \\
\hline \multicolumn{2}{|c|}{ *l'erreur standard $=\varepsilon=\sqrt{\frac{\varepsilon(x-\bar{x})^{2}}{n-1}}$} & \multicolumn{2}{|c|}{$\begin{aligned} x= & \text { valeur de } \Delta \frac{\mathrm{G}_{40}-\mathrm{G}_{10}}{\mathrm{G}_{10}} \times 100 \\
& \text { pour les différents essais. } \\
\bar{x}= & \text { valeur moyenne }=\frac{\varepsilon x}{\mathrm{n}} \\
& \text { n étant le nombre d'essais. }\end{aligned}$} \\
\hline
\end{tabular}

Il serait intéressant d'étendre l'application de cette méthode à d'autres fabrications fromagères en vue de déterminer les paramètres conductimétriques propres à chacune d'elles et de vérifier s'il existe une analogie dans la réponse avec ce que nous avons trouvé dans le présent travail.

Dans ces conditions la méthode conductimétrique pourrait apporter des solutions appréciables dans la fabrication des fromages.

\section{Rés u m é}

Les différentes phases de préparation du caillé en vue de l'élaboration du fromage Emmenthal à partir de lait frais de mélange sont suivies par la méthode conductimétrique.

L'enregistrement de l'évolution de la conductance du milieu permettrait en particulier de déterminer le temps optimal au bout duquel on procéderait à l'emprésurage après action des ferments lactiques et indiquerait au fromager le moment où il doit entrepren- 
dre les opérations suivant la formation du caillé quand ce dernier a atteint la texture désirée.

L'application de cette méthode à d'autres fabrications fromagères aiderait à définir les paramètres conductimétriques propres à chacune d'elles et apporterait des solutions non négligeables à l'industrie laitière.

\section{S u m m a r y}

The Conductrimetric method is used to realize the various phases for the curd-preparation in order to make Emmenthal cheese from fresh mixed milk.

To record the evolution of the medium-conductance would more particulary enable to determine the optimum - time at which rennet would have to be added after action of Lactic ferments and thus would indicate to the cheese - maker the time at which he has to Start the procedure after the curdled-mass has reached the wanted texture.

To apply this method to other cheese makings might enable to define the conductimetric parameters relevant to each of them and bring satiffactory solutions to the dairy industry.

Reçu pour publication en mai 1976.

\section{Bibliographie}

[1] Tsouli (J.), Favre-Bonvin (G.), Policard (C.) et Ville (A.) (1975). - Mesure par la méthode conductimétrique de l'activité enzymatique de trois variétés de présure sur du lait frais de mélange et du lait reconstitué à partir de poudre dégraissée. Le Lait, 545-546, 289-294.

[2] Policard (C.), Wallach (J.), Ville (A.) et Hanss (M.) (1974). - Ann. Phys. Biol. et Méd., vol. 8, $\mathrm{n}^{\circ} 1,17-23$.

[3] Policard (C.) (1972). - Thèse 3e cycle Biochimie, Lyon.

[4] Hanss (M.) et ReY (A.) (1971). - Biochimie Biophys. Acta, 227, 618-629 et 630-638.

[5] Hanss (M.), Dumontier (M.) et Policard (C.) (1975). - Spectra 2000, t. 3, $\mathrm{n}^{\circ} 16,33-38$.

[6] Alais (C.) (1975). - Science du Lait, 3e édition, sep., 162-189 et 641-661.

[7] Waugh (D. F.). - 2e Congrès Int. de Biophys., Vienne, 1966, Abst. 104, les An. de Phy. Biol et Méd. 